\title{
Bronchopleural fistula following intrauterine transfusion
}

\author{
H. V. PRICE, JOAN ANDREWS, and K. M. LAURENCE \\ Departments of Child Health and Obstetrics and Gynaecology, Welsh National School of Medicine, \\ Cardiff Maternity Hospital, Glossop Terrace, Cardiff
}

\begin{abstract}
An infant developed a bronchopleural fistula following intrauterine transfusion given for severe haemolytic disease of the newborn. The fistula was shown to be due to the transfusion being given into the right pleural cavity and lung. Initial treatment along conservative lines is suggested, reserving thoracotomy and lobectomy for use if these simple measures fail. Hypocalcaemia was a complicating feature.
\end{abstract}

The technique of intrauterine transfusion of the fetus in severe rhesus isoimmunization has enabled many infants to survive who would otherwise have died (Liley, 1963; Fairweather et al., 1967). Survival rates have so improved that 30 to $50 \%$ are now liveborn who would otherwise have died in utero. There are endless possibilities of injury to the fetus during the procedure yet surprisingly few have been reported.

This case is presented because of the previously unrecorded complication of bronchopleural fistula.

\section{CASE HISTORY}

This male infant was born to a healthy 41 -year-old mother in her fifth pregnancy. One previous child had required exchange transfusion for erythroblastosis fetalis. The father was heterozygous rhesus positive ( $\mathrm{CDe} / \mathrm{cde}$ ). The maternal antibody titre was positive at 1 in 256 dilution at 22 weeks' gestation. Amniocentesis at 26 weeks' gestation gives a high zone result (Liley, 1963).

The first intrauterine transfusion was carried out at 26 weeks' gestation. Several attempts were required before the fetal peritoneal cavity was punctured and $60 \mathrm{ml}$ of warmed packed blood infused. Spontaneous vaginal bleeding occurred the following day and lasted a few hours.

Ten days after the first intrauterine transfusion spontaneous rupture of the membranes occurred and liquor continued to drain thereafter. A second intrauterine transfusion was given during the twenty-eighth week, only one attempt at fetal puncture being required.

Labour began spontaneously in the thirtieth week following a small antepartum haemorrhage. A male infant weighing $1,400 \mathrm{~g}$ was delivered by the breech. The liver and spleen were both palpable $3 \mathrm{~cm}$ below the costal margin. The placenta weighed $350 \mathrm{~g}$ and was oedematous. The Apgar score was 1 at 1 minute rising to 6 at 10 minutes with the help of intermittent positive pressure ventilation and intravenous sodiumo bicarbonate and dextrose.

Cord blood results were: Coombs test positive haemoglobin $11.9 \mathrm{~g} / 100 \mathrm{ml}$, serum bilirubin $4 \mathrm{mg} \%$ $100 \mathrm{ml}$.

Small repeated exchange transfusions were given during the first day after birth, intravenous infusion of $10 \%$ dextrose being maintained in the intervening periods. The infant had moderately severe respiratory distress syndrome.

Ten hours after birth umbilical artery blood results were: haemoglobin $14.2 \mathrm{~g} / 100 \mathrm{ml}$, serum bilirubin $6.9 \mathrm{mg} / 100 \mathrm{ml}, \mathrm{pH} 6.9, \mathrm{PcO}_{2} 150 \mathrm{mmHg}$, base exces\$̊ $2 \mathrm{mEq} /$ litre, $\mathrm{PAO}_{2} 100 \mathrm{mmHg}$.

The infant was thus unable to maintain adequate ventilation in spite of adequate oxygenation and haof several brief apnoeic episodes.

Artificial ventilation with an Amsterdam ventilatoo was used from this time with maximum gauge in? flation pressures of $30 \mathrm{~cm}$ water. Twenty-two hour after birth the infant suddenly became cyanosed ancE. radiography confirmed a right tension pneumothorax

An underwater seal drain was inserted with im= provement but even with suction applied it was neve possible to inflate the lung more than half normal N A bronchogram was performed four days after birtlo but failed to demonstrate the suspected broncho pleural fistula. Seventy-two hours after birth umbilica? arterial blood results were: $\mathrm{pH} 7 \cdot 28, \mathrm{PcO}_{2} 57 \mathrm{mmHg}$ base deficit $1 \mathrm{mEq} /$ litre, $\mathrm{PAO}_{2} 90 \mathrm{mmHg}$, serum sodium 130 , potassium $3.7 \mathrm{mEq} / 1$, urea 90 , mago nesium 1.5 , calcium $3.9 \mathrm{mg} / 100 \mathrm{ml}$.

The infant was thus being fairly adequately ventio lated in spite of the pneumothorax. The hypocal $\mathbb{R}$ caemia was corrected with intravenous and oral supe plements of calcium gluconate. 


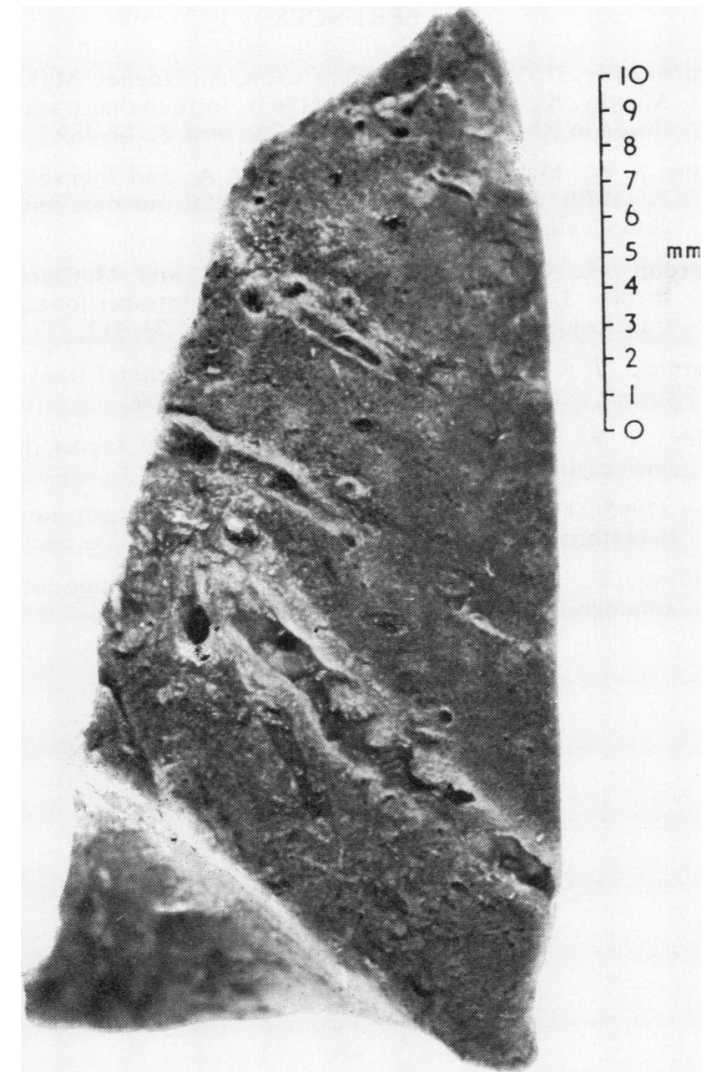

FIGURE. Cross-section of right lung to show track of bronchopleural fistula.

The infant gradually deteriorated thereafter and died seven days after birth.

At necropsy there was a large right-sided tension pneumothorax together with a small turbid icteric effusion, and haemosiderin staining of the pleural surfaces, whereas the left pleural cavity was normal. Both lungs were pneumonic but the right lung in addition showed some interstitial emphysema and there was a large bronchopleural fistula in the lower lobe (Figure) with old interstitial haemorrhage and haemosiderin-laden macrophages in the adjacent parenchyma.

In the abdomen the serous surfaces were also haemosiderin stained, and loops of bowel were adherent to one another. The enlarged icteric liver showed a needle track, fatty change, and bile retention. The enlarged spleen had many haemosiderin-laden macrophages and an area of infarction.

Other findings included a torn tentorium together with a large posterior and middle cranial fossae subdural haematoma, fatty change in the myocardium, thymic exhaustion, and, of course, widespread extramedullary haemopoiesis.

\section{DISCUSSION}

In spite of all the adverse features it seemed initially that the infant might survive with intensive care. The weakened area of the lung due to the misplaced intrauterine transfusion ruptured under the stress of intermittent positive pressure ventilation. It seems likely that the lung would have healed sufficiently firmly to withstand the stresses of artificial ventilation if delivery had been delayed for a few weeks. However, intraiterine transfusion is a recognized cause of premature labour. The mechanism is usually high rupture of the membranes by the intrauterine transfusion needle. The liquor amnii then tracks down between the uterus and membranes to the vagina and, if persistent, will produce premature labour. This was probably the cause of the premature labour in this infant.

Fairweather et al. (1967) report entering the pleural cavity on two occasions without any adverse effect, and with subsequent fetal survival.

Misplaced Tuohy needle punctures of the fetal spleen, heart, liver, bladder, gut, and even skull have been recorded (Liley, 1965; Fairweather et al., 1967; Karnicki, 1968). Liver haemorrhage resulting from paracentesis abdominis after birth has been recorded, but most liver punctures in utero heal spontaneously, as occurred in this infant (Gordon et al., 1966). The blood may be transfused into the abdominal wall and produce excessive jaundice after birth (Fong, Margolis, Westberg, and Johnson, 1970). Misplaced intrauterine injection of sodium diatrizoate (Renografin) into the forearm has produced sloughing of the skin (Sullivan and Smith, 1970). Sudden death may occur when the peritoneal cavity is entered, particularly in hydropic infants (Fong et al., 1970). Thus intrauterine transfusion has many hazards which can only be reduced by the high degree of technical skill acquired with frequent practice.

There seems little doubt that the bronchopleural fistula was caused by a misplaced intrauterine transfusion. The icteric pleural effusion and haemosiderin-stained pleural surfaces provide evidence of blood transfusion into the right pleural cavity. The possibility that the fistula was caused by the cannula used to evacuate the pneumothorax is unlikely. The cannula was inserted anteriorly through the second right intercostal space when the lung was collapsed and was not in line with the fistula track. Another explanation is that the soft suction cannula used for bronchial toilet during assisted ventilation was pushed right through the bronchial wall and lung substance. This is unlikely as we have never found evidence of rupture of the bronchial wall in over one hundred infants 
similarly treated with prolonged intermittent positive pressure ventilation.

Pneumothorax occurs in about $1 \%$ of all newborn infants but is usually asymptomatic. Symptomatic neonatal pneumothorax is most easily treated by insertion of a soft plastic catheter through a needle into the pleural space, for example, Portex or Intracath devices. The catheter is connected to an underwater seal drain to which suction can be applied. The rate of absorption of entrapped air is hastened with a high ambient oxygen concentration, and this is particularly helpful when there is coexistent pneumomediastinum or interstitial emphysema. In this infant these simple measures failed to produce improvement after several days. This leads us to suggest thoracotomy and lobectomy as one method of treatment in these circumstances.

\section{REFERENCES}

Fairweather, D. V. I., Tacchi, D., Coxon, A., Hughes, M. I., Murray, S., and Walker, W. (1967). Intrauterine trans-으 fusion in $\mathrm{Rh}$-isoimmunization. Brit. med.J., 14, 189.

Fong, S. W., Margolis, A. J., Westberg, J. A., and Johnson, P. (1970). Intrauterine transfusion; fetal outcome and complications. Pediatrics, 45, 576.

Gordon, H., Grausz, J. P., Raphael, M., and McClure. Brown, J. C. (1966). Experiences with intraperitoneal $\overrightarrow{+}$ transfusion. J. Obstet. Gynaec. Brit. Cwlth, 73, 917. Karnicki, J. (1968). Results and hazards of prenatal trans-
fusion. J. Obstet. Gynaec. Brit. Cwlth, 75, 1209.

Liley, A. W. (1963). Intrauterine transfusion of foetus in haemolytic disease. Brit. med. J., 2, 1107. (1965). The use of amniocentesis and foetal transfusion in erythroblastosis fetalis. Pediatrics, 35, 836.

Sullivan, T., and Smith, G. F. (1970). Complication of amniography. Lancet, 1, 946. 